\title{
Type II string theory on Calabi-Yau manifolds with torsion and non-Abelian discrete gauge symmetries
}

\author{
Volker Braun, ${ }^{a}$ Mirjam Cvetič, ${ }^{b, c, d}$ Ron Donagi ${ }^{c, b}$ and Maximilian Poretschkin ${ }^{b}$ \\ ${ }^{a}$ Elsenstrasse 35, 12435 Berlin, Germany \\ ${ }^{b}$ Department of Physics and Astronomy, University of Pennsylvania, \\ 209 S 33rd St, Philadelphia, PA 19104, U.S.A. \\ ${ }^{c}$ Department of Mathematics, University of Pennsylvania, \\ 209 S 33rd St, Philadelphia, PA 19104, U.S.A. \\ ${ }^{d}$ Center for Applied Mathematics and Theoretical Physics, University of Maribor, \\ Mladinska ulica 3, 2000 Maribor, Slovenia \\ E-mail: vbraun@map.cc, cvetic@physics.upenn.edu, \\ donagi@math. upenn. edu, mporet@sas.upenn.edu
}

ABSTRACT: We provide the first explicit example of Type IIB string theory compactification on a globally defined Calabi-Yau threefold with torsion which results in a fourdimensional effective theory with a non-Abelian discrete gauge symmetry. Our example is based on a particular Calabi-Yau manifold, the quotient of a product of three elliptic curves by a fixed point free action of $\mathbb{Z}_{2} \times \mathbb{Z}_{2}$. Its cohomology contains torsion classes in various degrees. The main technical novelty is in determining the multiplicative structure of the (torsion part of) the cohomology ring, and in particular showing that the cup product of second cohomology torsion elements goes non-trivially to the fourth cohomology. This specifies a non-Abelian, Heisenberg-type discrete symmetry group of the four-dimensional theory.

Keywords: String Field Theory, Conformal Field Models in String Theory, Discrete Symmetries

ArXiv EPrint: 1702.08071 


\section{Contents}

1 Introduction 1

2 Non-Abelian discrete symmetries in Type IIB 3

2.1 Generalizations 6

$\begin{array}{lll}3 & \text { The Calabi-Yau manifold } & 7\end{array}$

4 Submanifolds $\quad 8$

4.1 The special Lagrangian submanifold $Y \quad 9$

$\begin{array}{lll}4.2 & \text { The four-dimensional submanifold } Y_{0} & 11\end{array}$

4.3 Cohomology of the Calabi-Yau manifold 13

5 Cup product 13

$\begin{array}{lll}5.1 & \text { Cup product on } Y_{0} & 13\end{array}$

$\begin{array}{lll}5.2 & \text { Naturality and the Calabi Yau manifold } & 14\end{array}$

6 Outlook 14

$\begin{array}{ll}\text { A Notebook } & \mathbf{1 5}\end{array}$

$\begin{array}{ll}\text { A.1 Constructing } Y_{0} & 16\end{array}$

A.2 Cohomology groups: the fundamental region 17

$\begin{array}{ll}\text { A.3 Cohomology groups: four-torus } & 17\end{array}$

$\begin{array}{lll}\text { A.4 Cohomology groups of } Y_{0} & 18\end{array}$

$\begin{array}{lll}\text { A.5 Cup products } & 18\end{array}$

$\begin{array}{lll}\text { A.6 } & \text { Alternate basis } & 20\end{array}$

\section{Introduction}

Discrete symmetries are an integral part of particle physics; they play a key rôle in the Standard Model physics, such as those to prevent nucleon and lepton number violating processes, with R-symmetry being the most prominent one, as well as examples of Abelian and non-Abelian discrete symmetries to address the flavor hierarchy.

In string theory, discrete symmetries are expected to be gauge symmetries. In recent years there has been a flurry of activities in studies of discrete symmetries in string and Ftheory compactification, focusing on their geometric origin. In F-theory the primary focus was on the origin of Abelian discrete symmetries, which arise from Calabi-Yau geometries which are genus-one fibrations without section, in contrast to elliptic fibrations with sections. A natural object associated with these compactifications is the Tate-Shafarevich (TS) group which is a discrete Abelian group that organizes inequivalent genus-one geometries which share the same associated Jacobian fibration. The TS group specifies the Abelian discrete gauge symmetry of the F-theory compactification. The study of F-theory 
compactifications with discrete gauge symmetries $\mathbb{Z}_{n}$ was initiated in [1] and followed-up in [2-9]. Most of the past works primarily focused on $\mathbb{Z}_{2}$ gauge symmetry. However, new insights into aspects of the TS group, and its relations to M-theory vacua in the case of $\mathbb{Z}_{3}$ were addressed in [8]. Furthermore, there has been progress in elucidating the origin of Abelian discrete symmetries via F-theory/Heterotic duality [9].

On the other hand, the origin of non-Abelian discrete gauge symmetries in string theory is less understood. An important progress in this direction was made [10] in the context of Type II string theory compactification to four-dimensions, building on earlier works [11-13] that study Abelian discrete gauge symmetries in Type II string theory compactification on Calabi-Yau threefolds with torsion. In the context of Type IIB string theory, a nonAbelian Heisenberg-type discrete symmetry is realized [10] on a Calabi-Yau threefold with torsion whose second cohomology torsion elements have a non-trivial cup product into fourth cohomology torsion ones. This specific approach therefore requires the study of Calabi-Yau threefolds with torsion by determining torsion cohomology groups and their cup products, which is technically challenging.

The purpose of this paper is to provide a first explicit construction of a Type IIB string theory compactification on a Calabi-Yau manifold that exhibits a Heisenberg-type discrete symmetry. ${ }^{1}$ For that purpose we choose an example of a Calabi-Yau threefold $X_{6}$ with torsion. This particular Calabi-Yau threefold occurs in the classification of [15] as the first example of the free quotient of a torus $T^{6}$ by a finite group action. It had occurred previously, in various contexts, in [16-18]. It is the quotient of a product of three elliptic curves by a fixed point free action of $\mathbb{Z}_{2} \times \mathbb{Z}_{2}$ which we describe explicitly in (3.1). The integer cohomology of our Calabi-Yau manifold $X_{6}$ is given in (4.20). This Calabi-Yau has a symmetric Hodge diamond, with $h^{1,2}=h^{1,1}=3$. If it is also a self-mirror manifold, to the best of our knowledge, this would be the first example of a self-mirror Calabi-Yau threefold where the two (a priori independent) torsion groups Torsion $\left(H^{2}\left(X_{6}, \mathbb{Z}\right)\right)=\operatorname{Torsion}\left(H^{5}\left(X_{6}, \mathbb{Z}\right)\right)=$ $\mathbb{Z}_{4}^{2} \oplus \mathbb{Z}_{2}^{3}$ and Torsion $\left(H^{3}\left(X_{6}, \mathbb{Z}\right)\right)=$ Torsion $\left(H^{4}\left(X_{6}, \mathbb{Z}\right)\right)=\mathbb{Z}_{2}^{3}$ actually differ and are both non-zero.

This example allows us to carry out the challenging computation of the cup products for torsion coholomology elements explicitly, which in turn determine the Heisenberg discrete symmetry of the four-dimensional theory. The key strategy is to relate $X_{6}$ to a lower dimensional (real) submanifold $Y_{0}$. Recalling that $X_{6}$ is the quotient of a six-torus by a group $G=\mathbb{Z}_{2} \times \mathbb{Z}_{2}$, we take $Y_{0}$ to be the quotient of a four-dimensional subtorus of $X_{6}$ that happens to be invariant under $G$. It comes with an inclusion map $i: Y_{0} \rightarrow X_{6}$ and a projection map going the other way, hence the restriction $i^{*}: H^{*}\left(X_{6}, \mathbb{Z}\right) \rightarrow H^{*}\left(Y_{0}, \mathbb{Z}\right)$ is surjective, and in fact it exhibits $H^{*}\left(Y_{0}, \mathbb{Z}\right)$ as a direct summand of $H^{*}\left(X_{6}, \mathbb{Z}\right)$. The cup product calculation on the smaller $Y_{0}$ can be carried out explicitly. This could probably be done by hand using elementary topology. Instead, we obtain it as part of a computational scheme that gives us many additional useful facts about the manifolds involved, including the integer cohomology of $X_{6}$, given in (4.20).

\footnotetext{
${ }^{1}$ Non-Abelian discrete symmetries of Type IIB string theory on $A d S_{5} \times S^{5} / \mathbb{Z}_{3}$ were studied in [14] from the perspective of AdS/CFT correspondence.
} 
The cup product on $Y_{0}$ turns out to have the property we want: there are torsion classes in $H^{2}\left(Y_{0}, \mathbb{Z}\right)$ whose product does not vanish in $H^{4}\left(Y_{0}, \mathbb{Z}\right)$. Even though we do not fully calculate the multiplicative structure of the cohomlogy ring of $\mathrm{X}$, the fact that $H^{*}\left(Y_{0}, \mathbb{Z}\right)$ is a direct summand of $H^{*}\left(X_{6}, \mathbb{Z}\right)$ plus knowing the multiplicative structure of the cohomlogy ring of $Y_{0}$ suffices to allow us to conclude that there are torsion classes in $H^{2}\left(X_{6}, \mathbb{Z}\right)$ whose product does not vanish in $H^{4}\left(X_{6}, \mathbb{Z}\right)$, as claimed. In particular, Type IIB compactification on such a Calabi-Yau manifold leads to a four-dimensional theory with a Heisenberg-type discrete symmetry group. ${ }^{2}$

In section 2 we summarize the results of [10] regarding Type IIB string theory compactifications leading to four-dimensionall theories with Heisenberg-type discrete symmetries. Our Calabi-Yau manifold is described in section 3. The rest of the paper is devoted to the calculation of the torsion homologies and cup products for this manifold. In section 4 we determine the torsion cohomology groups for the Calabi-Yau manifold and some of its submanifolds, and in section 5 the specific results for the cup products are obtained. The outlook is given in section 6 . In the appendix we display the notebook containing the main calculations. A link is provided to a site containing the code and the worksheet, so that the interested reader can verify the calculations directly.

\section{Non-Abelian discrete symmetries in Type IIB}

In this section, we review the construction of non-Abelian discrete symmetries arising in Type IIB compactifications on a Calabi-Yau manifold $X_{6}$. The analysis follows closely that of [10]. This latter analysis is closely related to the study [11] of Abelian discrete symmetries in Type IIB compactifications.

In general, the homology of a threefold $X_{6}$ has two independent torsion sub-groups given by

$$
\begin{aligned}
& \operatorname{Torsion}\left(H_{1}\left(X_{6}, \mathbb{Z}\right)\right) \simeq \operatorname{Torsion}\left(H_{4}\left(X_{6}, \mathbb{Z}\right)\right), \\
& \operatorname{Torsion}\left(H_{2}\left(X_{6}, \mathbb{Z}\right)\right) \simeq \operatorname{Torsion}\left(H_{3}\left(X_{6}, \mathbb{Z}\right)\right),
\end{aligned}
$$

associated with the torsion one-cycles (and Poincaré dual torsion four-cycles) and torsion two-cycles (and Poincaré dual torsion three-cycles), respectively.

In the following, we shall first restrict our discussion to the case that

$$
\begin{aligned}
& \operatorname{Torsion}\left(H_{1}\left(X_{6}, \mathbb{Z}\right)\right) \simeq \operatorname{Torsion}\left(H_{4}\left(X_{6}, \mathbb{Z}\right)\right)=\mathbb{Z}_{k}, \\
& \operatorname{Torsion}\left(H_{2}\left(X_{6}, \mathbb{Z}\right)\right) \simeq \operatorname{Torsion}\left(H_{3}\left(X_{6}, \mathbb{Z}\right)\right)=\mathbb{Z}_{k^{\prime}} .
\end{aligned}
$$

The Poincaré dual cohomology groups which are needed for the dimensional reduction of Ramond-Ramond fields are accordingly given as

$$
\begin{aligned}
& \operatorname{Torsion}\left(H^{5}\left(X_{6}, \mathbb{Z}\right)\right) \simeq \operatorname{Torsion}\left(H^{2}\left(X_{6}, \mathbb{Z}\right)\right)=\mathbb{Z}_{k}, \\
& \operatorname{Torsion}\left(H^{4}\left(X_{6}, \mathbb{Z}\right)\right) \simeq \operatorname{Torsion}\left(H^{3}\left(X_{6}, \mathbb{Z}\right)\right)=\mathbb{Z}_{k^{\prime}} .
\end{aligned}
$$

\footnotetext{
${ }^{2}$ For a related work in the context of F-theory, see [19] where the origin of the Heisenberg-type discrete symmetry does not seem to have a weak coupling limit to Type IIB string theory. In the context of Heterotic string compactifications some aspects of torsion homologies were studied, e.g., in [20] for the Schoen manifold.
} 
Let $\rho_{2}, \beta_{3}, \tilde{\omega}_{4}$, and $\zeta_{5}$ represent the generators of the torsion cohomologies Torsion $\left(H^{2}\left(X_{6}, \mathbb{Z}\right)\right)$, Torsion $\left(H^{3}\left(X_{6}, \mathbb{Z}\right)\right)$, Torsion $\left(H^{4}\left(X_{6}, \mathbb{Z}\right)\right)$ and Torsion $\left(H^{5}\left(X_{6}, \mathbb{Z}\right)\right)$, respectively. They satisfy the following relations

$$
\begin{array}{rlrl}
\delta \gamma_{1} & =k \rho_{2}, & \delta \tilde{\rho}_{4}=k \zeta_{5}, \\
\delta \alpha_{3}=k^{\prime} \tilde{\omega}_{4}, & \delta \omega_{2}=k^{\prime} \beta_{3},
\end{array}
$$

where $\gamma_{1}, \omega_{2}, \alpha_{3}$ and $\tilde{\rho}_{4}$ are one-, two-, three- and four- cochains on $X_{6}$, respectively, belonging to corresponding $H^{*}\left(X_{6}, \mathrm{U}(1)\right)$ cohomologies. ${ }^{3}$ The above elements satisfy:

$$
\int_{X_{6}} \gamma_{1} \wedge \zeta_{5}=\int_{X_{6}} \rho_{2} \wedge \tilde{\rho}_{4}=\int_{X_{6}} \alpha_{3} \wedge \beta_{3}=\int_{X_{6}} \omega_{2} \wedge \tilde{\omega}_{4}=1 .
$$

Here $k^{-1}$ and $k^{\prime-1}$ are the torsion linking numbers between dual torsion $p$ - and $(5-p)$ cycles $(p=1,3)$. Note, eqs. (2.4) and (2.5) can be obtained from expressions that determine torsion linking numbers, cf., appendix $\mathrm{C}$ of [11].

The cup-product of two torsion classes is again a torsion class. Thus the product $\rho_{2} \wedge \rho_{2}$ is some multiple of the generator $\tilde{\omega}_{4}$ of Torsion $\left(H^{4}\left(X_{6}, \mathbb{Z}\right)\right)$ :

$$
\rho_{2} \wedge \rho_{2}=M \tilde{\omega}_{4}, \quad M \in \mathbb{Z} .
$$

The coefficient $M$ is an invariant of the manifold $X_{6}$. Sometimes it vanishes, and sometimes it does not. In this work we describe an example where it is non zero. By employing (2.4) this cup-product integrates to $\rho_{2} \wedge \gamma_{1}=M^{\prime} \alpha_{3}$, where $M^{\prime} \in \mathbb{Z}$ and $k M=k^{\prime} M^{\prime}$.

These torsion subgroups give a priori rise to three non-commuting discrete cyclic groups in the effective four-dimensional Type IIB action. This can be seen from the following Kaluza-Klein reduction Ansatz for the Type IIB closed string sector Ramond-Ramond (RR) and Neveu-Schwarz-Neveu-Schwarz (NSNS) two-form fields $C_{2}, B_{2}$, respectively and RR four-form field $C_{4}$ :

$$
\begin{aligned}
& B_{2}=b^{1} \wedge \rho_{2}+A^{1} \wedge \gamma_{1}, \\
& C_{2}=b^{2} \wedge \rho_{2}+A^{2} \wedge \gamma_{1}, \\
& C_{4}=b^{3} \tilde{\omega}_{4}+A^{3} \wedge \alpha_{3}+V^{3} \wedge \beta_{3}+c_{2} \wedge \omega_{2},
\end{aligned}
$$

where $b^{i}$ and $A^{i}(i=1,2,3)$ are the three axions and three $\mathrm{U}(1)$ one-form gauge potentials, respectively. (One-form potential $V^{3}$ and two-form potential $c_{2}$, are not independent fields, due to the self-duality of the five-form field strength $F_{5}$ in Type IIB supergravity.)

These Ansätze ensure that the Kaluza-Klein reduction of Type IIB supergravity results in an effective four-dimensional field theory with three massive $\mathrm{U}(1)$ one-form gauge potentials $A^{i}(=i=1,2,3)$. (For further details, see section 4 of [10].) E.g., for $B_{2}$ one obtains:

$$
\begin{aligned}
& \int_{M_{10}=M_{4} \times X_{6}} d B_{2} \wedge * d B_{2} \longrightarrow \\
& \int_{M_{4}}\left(d b^{1}-k A^{1}\right) \wedge *\left(d b^{1}-k A^{1}\right) \int_{X_{6}} \rho_{2} \wedge * \rho_{2}+\int_{M_{4}}\left(d A^{1}\right) \wedge *\left(d A^{1}\right) \int_{X_{6}} \gamma_{1} \wedge * \gamma_{1},
\end{aligned}
$$

\footnotetext{
${ }^{3}$ This approach is a simplified version designed to keep the expository part short and clear. A better mathematical treatment would involve Cheeger-Simons cocycles or, more or less equivalently, Deligne cohomology. These keep track of both the continuous and the discrete parameters.
} 
which results in a Stückelberg mass term for $A^{1}$. The Stückelberg mass contributions for all three gauge fields $A^{1}, A^{2}, A^{3}$ in the effective four-dimensional action is of the following schematic form:

$$
\mathcal{L} \supset \mathcal{G}_{i j} \eta_{\mu}^{i} \eta^{\mu j}
$$

where

$$
\begin{aligned}
& \eta_{\mu}^{i}=\partial_{\mu} b^{i}-k A_{\mu}^{i}, \quad i=1,2, \\
& \eta_{\mu}^{3}=\partial_{\mu} b^{3}-k^{\prime} A_{\mu}^{3}-M b^{2}\left(\partial_{\mu} b^{1}-k A_{\mu}^{1}\right) .
\end{aligned}
$$

This four-dimensional action is therefore invariant under the following non-commuting discrete gauge transformations:

$$
\begin{array}{ll}
A_{\mu}^{i} \rightarrow A_{\mu}^{i}+\partial_{\mu} \lambda^{i}, & b^{i} \rightarrow b^{1}+k \lambda^{i}, \quad i=1,2, \\
A_{\mu}^{3} \rightarrow A_{\mu}^{3}+\partial_{\mu} \lambda^{3}+M^{\prime} k \lambda^{2} A_{\mu}^{1}+M^{\prime} b^{1} \partial_{\mu} \lambda^{2}, & b^{3} \rightarrow b^{3}+M k b^{1} \lambda^{2}+k^{\prime} \lambda^{3},
\end{array}
$$

where $M \in \mathbb{Z}, M^{\prime} \in \mathbb{Z}$ and $k M=k^{\prime} M^{\prime}$. This corresponds to a set of non-commuting $\mathbb{Z}_{k}$, $\mathbb{Z}_{k}, \mathbb{Z}_{k^{\prime}}$ factors as long as $M \neq 0$, resulting in a non-Abelian discrete gauge symmetry of the four-dimensional action, specified by $k, k^{\prime}$ and $M$.

Altogether there are three generators $T_{1}, T_{2}, T_{3}$ associated with the discrete symmetry groups $\mathbb{Z}_{k}, \mathbb{Z}_{k}, \mathbb{Z}_{k^{\prime}}$, respectively. The important fact to note is that these generators $T_{1}, T_{2}, T_{3}$ do not commute, provided that there is a non-trivial cup-product (2.6).

These discrete gauge symmetries of the effective four-dimensional action lead to the following discrete symmetry operations on a four-dimensional state $\psi(x)$, with charges $\left(q_{1}, q_{2}, q_{3}\right)$ under $\left(\mathbb{Z}_{k}, \mathbb{Z}_{k}, \mathbb{Z}_{k^{\prime}}\right)$. From a ten-dimensional perspective this state corresponds to a bound state of $q_{1}$ fundamental strings and $q_{2}$ D1-branes wrapping the torsional onecycle in Torsion $\left(H_{1}\left(X_{6}, \mathbb{Z}\right)\right)=\mathbb{Z}_{k}$, and $q_{3}$ D3-branes wrapping the torsional three-cycle in Torsion $\left(H_{3}\left(X_{6}, \mathbb{Z}\right)\right)=\mathbb{Z}_{k^{\prime}}{ }^{4}$ The state transforms under $T_{1}, T_{2}, T_{3}$ generators:

$$
\begin{aligned}
& \tilde{T}_{1}: \psi(x) \longrightarrow e^{2 \pi i k^{-1} q_{1}} \psi(x), \\
& \tilde{T}_{2}: \psi(x) \longrightarrow e^{2 \pi i k^{-1} q_{2}} \mathcal{U} \psi(x), \\
& \tilde{T}_{3}: \psi(x) \longrightarrow e^{2 \pi i k^{\prime-1} q_{3}} \psi(x),
\end{aligned}
$$

where the charge redefinition matrix $\mathcal{U}$ is of the form:

$$
\left(\begin{array}{l}
q_{1} \\
q_{2} \\
q_{3}
\end{array}\right) \mapsto\left(\begin{array}{ccc}
1 & 0 & M^{\prime} \\
0 & 1 & 0 \\
0 & 0 & 1
\end{array}\right)\left(\begin{array}{l}
q_{1} \\
q_{2} \\
q_{3}
\end{array}\right), \quad k M=k^{\prime} M^{\prime} .
$$

\footnotetext{
${ }^{4}$ General $\left(q_{1}, q_{2}\right)$-strings, which source $B_{2}$ and $C_{2}$ gauge potentials, wrap torsional one-cycles and result in a $\mathbb{Z}_{k} \times \mathbb{Z}_{k}$ symmetry at the level of four-dimensional particle states. D3-branes, which source $C_{4}$ gauge potential, wrap torsional three-cycles and result in an additional $\mathbb{Z}_{k^{\prime}}$ symmetry factor. When the corresponding torsional classes have non-trivial relations, as spelled out above, this Abelian structure is promoted to a non-Abelian one. This phenomenon is analogous to the Hanany-Witten effect [21] where fourdimensional strings emerge from NS5-branes and D5-branes wrapping torsional four-cycles, and D3-branes wrapping torsional two-cycles. The non-Abelian discrete symmetry emerges when torsional four-cycles intersect non-trivially along a torsional two-cycle; crossing of four-dimensional strings, associated with NS5and D5-branes, leads to the creation of strings associated with D3-branes wrapping torsional two-cycles.
} 
These transformations can be derived from the ten-dimensional perspective by performing discrete shift transformations on corresponding gauge potentials, supporting $p$-branes, and deducing the induced charges after the discrete shifts in the ten-dimensional Chern-Simons terms of the effective action. (Again, for further details see [10].)

Thus, one observes that

$$
\tilde{T}_{1} \tilde{T}_{2}=\tilde{T}_{3}^{M} \tilde{T}_{2} \tilde{T}_{1}
$$

resulting in a non-commuting discrete gauge symmetry, a Heisenberg discrete symmetry group $\left(\mathbb{Z}_{k} \times \mathbb{Z}_{k^{\prime}}\right) \rtimes \mathbb{Z}_{k}$, specified by $k, k^{\prime}$ and $M$. In special cases, say, when $k=k^{\prime}$, $M=1$ the non-Abelian discrete gauge symmetry is given by $\left(\mathbb{Z}_{k} \times \mathbb{Z}_{k}\right) \rtimes \mathbb{Z}_{k}$ and further specializations of $k$ reduce to, e.g., $D i h_{4}$ for $k=2$ and $\Delta(27)$ for $k=3$.

\subsection{Generalizations}

It is straightforward to generalize this analysis to the case when the second torsion cohomologies have multiple discrete factors. (For further details, see [19], section 2.)

Let us focus on the following specific examples, which shall be relevant for the rest of our analysis. The torsion cohomologies are chosen to be:

$$
\begin{aligned}
& \operatorname{Torsion}\left(H^{5}\left(X_{6}, \mathbb{Z}\right)\right) \simeq \operatorname{Torsion}\left(H^{2}\left(X_{6}, \mathbb{Z}\right)\right)=\mathbb{Z}_{k_{1}} \times \mathbb{Z}_{k_{2}}, \\
& \operatorname{Torsion}\left(H^{4}\left(X_{6}, \mathbb{Z}\right)\right) \simeq \operatorname{Torsion}\left(H^{3}\left(X_{6}, \mathbb{Z}\right)\right)=\mathbb{Z}_{k_{3}},
\end{aligned}
$$

and the nontrivial cup product of the generators $\rho_{2}^{(i)} \in \mathbb{Z}_{k_{i}}(i=1,2)$ is of the following form:

$$
\rho_{2}^{(1)} \wedge \rho_{2}^{(2)}=M \tilde{\omega}_{4},
$$

where $\tilde{\omega}_{4}$ is the generator of Torsion $\left(H^{4}\left(X_{6}, \mathbb{Z}\right)\right)=\mathbb{Z}_{k_{3}}$.

These generators satisfy the following relations: ${ }^{5}$

$$
\begin{aligned}
\delta \gamma_{1}^{(i)} & =k_{i} \rho_{2}^{(i)}, & \delta \tilde{\rho}_{4(i)} & =k_{i} \zeta_{5(i)}, \quad i=1,2, \\
\delta \alpha_{3} & =k_{3} \tilde{\omega}_{4}, & \delta \omega_{2} & =k_{3} \beta_{3},
\end{aligned}
$$

where $\beta_{3}$, and $\zeta_{5(i)}(i=1,2)$ represent the generators of the torsion cohomologies Torsion $\left(H^{4}\left(X_{6}, \mathbb{Z}\right)\right)$ and Torsion $\left(H^{5}\left(X_{6}, \mathbb{Z}\right)\right)$, respectively, and $\gamma_{1(i)}, \omega_{2}, \alpha_{3}$ and $\tilde{\rho}_{4(i)}$ are respective one-, two-, three- and four- cochains, elements of the corresponding $H^{*}\left(X_{6}, \mathrm{U}(1)\right)$ cohomologies, that satisfy:

$$
\int_{X_{6}} \gamma_{1}^{(i)} \wedge \zeta_{5(j)}=\int_{X_{6}} \rho_{2}^{(i)} \wedge \tilde{\rho}_{4(j)}=\delta_{j}^{i}, \quad \int_{X_{6}} \alpha_{3} \wedge \beta_{3}=\int_{X_{6}} \omega_{2} \wedge \tilde{\omega}_{4}=1 .
$$

Kaluza-Klein Ansätze for $B_{2}, C_{2}$ and $C_{4}$ gauge potentials, parallel those of (2.9):

$$
\begin{aligned}
& B_{2}=b_{(i)}^{1} \wedge \rho_{2}^{(i)}+A_{(i)}^{1} \wedge \gamma_{1}^{(i)}, \\
& C_{2}=b_{(i)}^{2} \wedge \rho_{2}^{(i)}+A_{(i)}^{2} \wedge \gamma_{1}^{(i)}, \\
& C_{4}=b^{3} \tilde{\omega}_{4}+A^{3} \wedge \alpha_{3}+V^{3} \wedge \beta_{3}+c_{2} \wedge \omega_{2} .
\end{aligned}
$$

\footnotetext{
${ }^{5}$ For the sake of simplicity, we chose specialized relations; for the analysis of more general cases, see [19].
} 
In the four-dimensional effective action there are five massive $\mathrm{U}(1)$ gauge fields $A_{(i)}^{1}, A_{(i)}^{2}$ and $A^{3}$, and five associated axions $b_{(i)}^{1}, b_{(i)}^{2}$, and $b^{3}$, respectively. (Again, $V^{3}$ and $c_{2}$ are not independent fields, due to the self-duality of $F_{5}$.)

The Stückelberg mass contributions for to the effective action again takes the schematic form:

$$
\mathcal{L} \supset \mathcal{G}_{I J *} \eta_{\mu}^{I} \eta^{\mu J^{*}}
$$

where $\eta_{\mu}^{I}$, complexified four-vectors, which take the following form:

$$
\begin{aligned}
\eta_{\mu(i)} & =\partial_{\mu} b_{(i)}^{2}-\tau \partial_{\mu} b_{(i)}^{1}+k_{i}\left(A_{\mu(i)}^{2}-\tau A_{\mu(i)}^{1}\right), \quad i=1,2, \\
\eta_{\mu}^{3} & =\partial_{\mu} b^{3}+k_{3} A_{\mu}^{3}-M\left(b_{(1)}^{2}-\tau b_{(1)}^{1}\right) k_{2} A_{\mu(2)}^{1}
\end{aligned}
$$

and $\tau=C_{0}+\mathrm{ie}^{-\phi}$ denotes the complexified string coupling of Type IIB string theory. This structure results in the discrete gauge invariance of the effective four-dimensional action, which corresponds to the Heisenberg discrete symmetry specified by $k_{1}, k_{2}, k_{3}$ and $M$. For further details see [10] section 2 and [19], section 3.

Thus, in order to determine the Heisenberg discrete group of Type IIB string compactifications on a Calabi-Yau threefold with torsion, the plan is to identify second cohomology torsion classes and to determine their non-trivial cup products. As explained in the introduction, we proceed to relate the Calabi-Yau threefold $X_{6}$ with torsion to a simpler space $Y_{0}$, a submanifold, where the cup product is under control. In particular, we exhibit a torsion class $t$ in the second cohomology $H^{2}\left(X_{6}, \mathbb{Z}\right)$ whose restriction to $Y_{0}$ is non-zero and squares to a non- zero class on the auxiliary $Y_{0}$. Functoriality of this cup product then fixes the rest. In this paper we apply this strategy the example of the Calabi-Yau threefold $X_{6}$, defined in the section below, and explicit calculations are derived in the subsequent two sections 4 and 5 .

\section{The Calabi-Yau manifold}

Our Calabi-Yau threefold $X,{ }^{6}$ will be the quotient of a six-torus (in fact the product of three elliptic curves) by a finite group action. The first and best known example of such a quotient was studied by Vafa and Witten in [22]. Let $E_{i}=\mathbb{C} /\left(\mathbb{Z}+\tau_{i} \mathbb{Z}\right) \ni z_{i}$, be three elliptic curves, $i=0,1,2$. Their product admits an action of the group $G=\mathbb{Z}_{2} \times \mathbb{Z}_{2}$, generated by the transformations;

$$
\begin{aligned}
& g_{1}^{0}:\left(z_{0}, z_{1}, z_{2}\right) \mapsto\left(z_{0},-z_{1},-z_{2}\right), \\
& g_{2}^{0}:\left(z_{0}, z_{1}, z_{2}\right) \mapsto\left(-z_{0}, z_{1},-z_{2}\right) .
\end{aligned}
$$

Vafa and Witten then consider a crepant resolution of this quotient.

Various other quotients, by different group actions, were considered by Oguiso and Sakurai [18] in the process of studying the collection of all Calabi-Yau manifolds with an infinite fundamental group. They obtained a partial classification, which has very recently

\footnotetext{
${ }^{6}$ For simplicity, in the rest of the paper we shall omit the subscript 6 for a Calabi-Yau threefold, i.e. $X_{6} \rightarrow X$.
} 
been completed in [23]. All actions of the basic group $G=\mathbb{Z}_{2} \times \mathbb{Z}_{2}$ and of all its Abelian extensions were classified in [15]. (Those actions of Abelian extensions that specialize to the Vafa-Witten action on $G$ had been classified earlier, in [24].) It turns out that, up to obvious equivalences, there are four such actions of $G$, cf. [15], Lemma 1.2.2. They all have the same linearization, so they differ only in the shifts. Exactly one of these $G$ actions is fixed-point free: the fixed-point free $G=\mathbb{Z}_{2} \times \mathbb{Z}_{2}$ action is generated by the transformations:

$$
\begin{aligned}
& g_{1}:\left(z_{0}, z_{1}, z_{2}\right) \mapsto\left(z_{0}+\frac{1}{2},-z_{1},-z_{2}\right), \\
& g_{2}:\left(z_{0}, z_{1}, z_{2}\right) \mapsto\left(-z_{0}, z_{1}+\frac{1}{2},-z_{2}+\frac{1}{2}\right) .
\end{aligned}
$$

This modifies the Vafa-Witten action by adding some non-trivial shifts. It is these shifts that make the action fixed-point free, and therefore the quotient:

$$
X=\left(E_{0} \times E_{1} \times E_{2}\right) / G
$$

is a manifold, with no need for a resolution. This particular quotient is described in example 2.17 of [18], where it is attributed to Igusa [16], page 678, and to Ueno [17], Example [16.16]. Each of $g_{1}, g_{2}$ and $g_{3}:=g_{1} \circ g_{2}$ preserves the constant holomorphic $(3,0)$ form on $E_{0} \times E_{1} \times E_{2}$, so this form descends to a nowhere vanishing holomorphic (3,0)-form on $X$. On the other hand, the $g_{i}$ project out any constant one-forms, so the quotient $X$ of (3.3) is a proper Calabi-Yau threefold in the sense that compactification preserves only the minimal amount of supersymmetry. We note that its holonomy group is $G$, and its fundamental group is easily seen to be the semidirect product

$$
\pi_{1}(X)=\mathbb{Z}^{6} \rtimes G,
$$

cf. [15], section 1.5 and table 1 on page 10. Finally, the only invariant $(1,1)$-forms are $d z_{i} \wedge d \bar{z}_{i}, i=0,1,2$, leading to the Hodge diamond

$$
\begin{aligned}
& 1 \\
& \begin{array}{ll}
0 & 0
\end{array}
\end{aligned}
$$

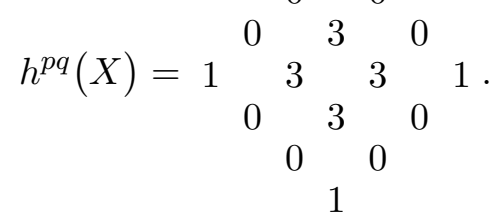

For further details see [15]. In this work we will analyze the cohomology ring of this Calabi-Yau manifold $X$ of (3.3). In [15] it was noted (Lemma 1.7.1) that this is one of four topologically inequivalent free quotients (the others are by various abelian extensions of $G)$. All are Calabi-Yau manifolds with Hodge numbers $(3,3)$, and it seems plausible that similar calculations can be carried out for each of these fixed-point free actions. We will not pursue these other manifolds in the present work.

\section{Submanifolds}

Since the group action eq. (3.2) only ever changes the imaginary part of the coordinates $z_{i}$ by a sign, there are a number of $G$-invariant (real) submanifolds of the product, hence 
submanifolds of the quotient $X$, obtained by setting the imaginary part to zero. On the covering space, these are sub-tori of $E_{0} \times E_{1} \times E_{2}$. After dividing out the group action, we obtain the special Langrangian 3-manifold

$$
Y \hookrightarrow X, \quad\left(x_{0}, x_{1}, x_{2}\right) \mapsto\left(x_{0}, x_{1}, x_{2}\right),
$$

three four-dimensional submanifolds

$$
\begin{aligned}
& Y_{0} \hookrightarrow X, \quad\left(x_{0}, x_{1}, x_{2}, y_{0}\right) \mapsto\left(x_{0}+\tau_{0} y_{0}, x_{1}, x_{2}\right), \\
& Y_{1} \hookrightarrow X, \quad\left(x_{0}, x_{1}, x_{2}, y_{1}\right) \mapsto\left(x_{0}, x_{1}+\tau_{1} y_{1}, x_{2}\right), \\
& Y_{2} \hookrightarrow X, \quad\left(x_{0}, x_{1}, x_{2}, y_{2}\right) \mapsto\left(x_{0}, x_{1}, x_{2}+\tau_{2} y_{2}\right),
\end{aligned}
$$

and three 5-dimensional submanifolds

$$
\begin{aligned}
& Y_{01} \hookrightarrow X, \quad\left(x_{0}, x_{1}, x_{2}, y_{0}, y_{1}\right) \mapsto\left(x_{0}+\tau y_{0}, x_{1}+\tau y_{1}, x_{2}\right), \\
& Y_{02} \hookrightarrow X, \quad\left(x_{0}, x_{1}, x_{2}, y_{0}, y_{2}\right) \mapsto\left(x_{0}+\tau y_{0}, x_{1}, x_{2}+\tau y_{2}\right), \\
& Y_{12} \hookrightarrow X, \quad\left(x_{0}, x_{1}, x_{2}, y_{1}, y_{2}\right) \mapsto\left(x_{0}, x_{1}+\tau y_{1}, x_{2}+\tau y_{2}\right) .
\end{aligned}
$$

In addition to the submanifold embeddings, we note that there are also projection maps $X \rightarrow Y_{i j}, X \rightarrow Y_{i}$, and $X \rightarrow Y$ by ignoring the imaginary part of one, two, and all three complex coordinates. Therefore, these submanifolds are all retractions and the relative cohomology long exact sequences split into

$$
\begin{aligned}
H^{*}(X, \mathbb{Z}) & \simeq H^{*}(Y, \mathbb{Z}) \oplus H^{*}(X, Y, \mathbb{Z}) & & \\
& \simeq H^{*}\left(Y_{i}, \mathbb{Z}\right) \oplus H^{*}\left(X, Y_{i}, \mathbb{Z}\right), & & 0 \leq i<3, \\
& \simeq H^{*}\left(Y_{i j}, \mathbb{Z}\right) \oplus H^{*}\left(X, Y_{i j}, \mathbb{Z}\right), & & 0 \leq i<j<3 .
\end{aligned}
$$

In the remainder of this section we now discuss the integral cohomology of these submanifolds.

\subsection{The special Lagrangian submanifold $Y$}

Again, all constant 2-forms on the covering space torus are projected out by the $G$-action. Hence, this is a rational homology sphere. Its fundamental group and Abelianization is

$$
\pi_{1}(Y)=\mathbb{Z}^{3} \rtimes G, \quad H_{1}(Y)=\pi_{1} /\left[\pi_{1}, \pi_{1}\right]=\mathbb{Z}_{4} \oplus \mathbb{Z}_{4} .
$$

To summarize, the integral cohomology is

$$
H^{d}(Y, \mathbb{Z})= \begin{cases}\mathbb{Z} & d=3 \\ \mathbb{Z}_{4} \oplus \mathbb{Z}_{4} & d=2 \\ 0 & d=1 \\ \mathbb{Z} & d=0\end{cases}
$$

and, by degree count, there can be no non-trivial cup products.

To get some explicit handle on the torsion cohomology generators, let us write down cochains in a cellular model. Our model of choice is going to be cubical cells with identifications. First, by cubical cells we mean (hyper-)cubes in some $\mathbb{R}^{n}$ whose vertices are 
integral, edges are parallel to the axes, and side lengths are either zero or one only. We write such a cube as

$$
\left[a_{0,0}, a_{0,1}\right] \times\left[a_{1,0}, a_{1,1}\right] \times \cdots \times\left[a_{n-1,0}, a_{n-1,1}\right]
$$

subject to the constraints

- $a_{k, l} \in \mathbb{Z}$ for all $0 \leq k<n, l \in\{0,1\}$,

- $a_{k, 0}=a_{k, 1}$ or $a_{k, 0}+1=a_{k, 1}$ for all $0 \leq k<n$.

To efficiently represent tori and quotients thereof, we also allow identifications of cubical cells, possibly with the opposite orientation. Hence, we will use equivalence classes of oriented cubical cells as our basic cell, which we will write as

$$
\left[a_{0,0}, a_{0,1}\right] \times\left[a_{1,0}, a_{1,1}\right] \times \cdots \times\left[a_{n-1,0}, a_{n-1,1}\right] / \sim
$$

and always refer to as cubical cells / cell complex in the following.

Although cubical cells are clearly convenient for describing tori, one might wonder whether the existence of a cubical cell complex imposes any constraint on a manifold. In fact, for topological manifolds we now known [25] that non-triangulable manifolds, that is, manifolds not homeomorphic to any simplicial complex, exist in each dimension $\geq 4$. However, for smooth manifolds such as ours, the situation is notably different, and triangulations always exist, by an old theorem of Whitehead. Finally, we note that the existence of a triangulation and of a cubical cell decomposition are equivalent. In one direction this equivalence is clear: the barycentric subdivision of any polyhedron (in particular, of a cube) yields a decomposition into simplices. Conversely, consider a $d$-simplex and its barycentric subdivision into $(d+1)$ ! top-dimensional simplices. For each of the original $d+1$ vertices, note that the $d$ ! adjacent sub-simplices fit together into a $d$-dimensional cube. In fact, this is the dual polyhedral decomposition of the simplex, and it defines a canonical cubical decomposition of a triangulation. Therefore, a smooth manifold always has a decomposition into a cubical cell complex.

Coming back to $Y$, we start with eight maximal cells ${ }^{7}$

$$
\begin{array}{ll}
{[0,1] \times[0,1] \times[0,1],} & {[0,1] \times[0,1] \times[1,2],} \\
{[0,1] \times[1,2] \times[0,1],} & {[0,1] \times[1,2] \times[1,2],} \\
{[1,2] \times[0,1] \times[0,1],} & {[1,2] \times[0,1] \times[1,2],} \\
{[1,2] \times[1,2] \times[0,1],} & {[1,2] \times[1,2] \times[1,2] .}
\end{array}
$$

By identifying opposite sides, this is a 3-torus with real coordinates

$$
\left(\xi_{0}, \xi_{1}, \xi_{2}\right)=\left(2 x_{0}, 2 x_{1}, 2 x_{2}\right) \in(\mathbb{R} / 2 \mathbb{Z})^{3},
$$

\footnotetext{
${ }^{7}$ Before any identifications, the cubical complex consists of 8 three-cubes, 36 squares, 54 line segments, and 27 vertices.
} 
and, using these coordinates, the $G$ action becomes (compare [15], page 6 and Lemma 1.2.2):

$$
\begin{aligned}
& g_{1}:\left(\xi_{0}, \xi_{1}, \xi_{2}\right) \mapsto\left(\xi_{0}+1,-\xi_{1},-\xi_{2}\right), \\
& g_{2}:\left(\xi_{0}, \xi_{1}, \xi_{2}\right) \mapsto\left(-\xi_{0}, \xi_{1}+1,-\xi_{2}+1\right) .
\end{aligned}
$$

The equivalence classes of cells under both the group action and identification of opposite sides in listed in table 1. Using this notation, the two generators of $H^{2}(Y, \mathbb{Z})=\mathbb{Z}_{4} \oplus \mathbb{Z}_{4}$ can be written as cochains

$$
\begin{aligned}
& \hat{c}_{1}=\chi([0,1] \times[0,1] \times[0,0] / \sim), \\
& \hat{c}_{2}=\chi([0,0] \times[0,1] \times[0,1] / \sim)+\chi([0,1] \times[0,0] \times[1,2] / \sim),
\end{aligned}
$$

where $\chi(c)$ denotes the cochain dual to the cell $c$, that is, the cochain that evaluates to one on $c$ and to zero on all other cells.

\subsection{The four-dimensional submanifold $Y_{0}$}

Note that $Y_{0}$ is not orientable, so its top cohomology group is $\mathbb{Z}_{2}$. The fundamental group and Abelianization of $Y_{0}$ is

$$
\pi_{1}\left(Y_{0}\right)=\mathbb{Z}^{4} \rtimes G, \quad H_{1}\left(Y_{0}\right)=\pi_{1} /\left[\pi_{1}, \pi_{1}\right]=\mathbb{Z}_{2} \oplus \mathbb{Z}_{4} \oplus \mathbb{Z}_{4} \oplus \mathbb{Z} .
$$

Finally, its degree-3 cohomology is not going to be relevant for even-degree cup products in the following, but can easily be determined numerically from the cell complex structure. To summarize, the integral cohomology is

$$
H^{d}\left(Y_{0}, \mathbb{Z}\right)= \begin{cases}\mathbb{Z}_{2} & d=4 \\ \mathbb{Z}^{2} & d=3 \\ \mathbb{Z}_{2} \oplus \mathbb{Z}_{4} \oplus \mathbb{Z}_{4} \oplus \mathbb{Z} & d=2 \\ 0 & d=1 \\ \mathbb{Z} & d=0 .\end{cases}
$$

We observe that the degrees are such that there can be a non-trivial cup product $H^{2} \times H^{2} \rightarrow$ $H^{4}$ involving torsion cohomology classes, which we will investigate in subsection 5.1.

The cubical complex for $Y_{0}$ is very similar to table 1 , the only change is that we add a factor $\times[0,1]$ for the $y_{0}$ coordinate, that is, use coordinates

$$
\left(\xi_{0}, \xi_{1}, \xi_{2}, \eta_{0}\right)=\left(2 x_{0}, 2 x_{1}, 2 x_{2}, y_{0}\right) \in(\mathbb{R} / 2 \mathbb{Z})^{3} \times(\mathbb{R} / \mathbb{Z}) .
$$

Note that the group action

$$
\begin{aligned}
& g_{1}:\left(\xi_{0}, \xi_{1}, \xi_{2}, \eta_{0}\right) \mapsto\left(\xi_{0}+1,-\xi_{1},-\xi_{2}, \eta_{0}\right), \\
& g_{2}:\left(\xi_{0}, \xi_{1}, \xi_{2}, \eta_{0}\right) \mapsto\left(-\xi_{0}, \xi_{1}+1,-\xi_{2}+1,-\eta_{0}\right) .
\end{aligned}
$$

never shifts $\eta_{0}$, which is why we do not need any subdivision in the cubical complex in that direction. After identifying opposing sides and $G$-images, we can again write down explicit 


\begin{tabular}{|c|c|c|c|}
\hline $\operatorname{dim}$ & cell & same orientation & opposite orientation \\
\hline 3 & {$[0,1] \times[0,1] \times[0,1] / \sim$} & $\begin{array}{r}\{[0,1] \times[0,1] \times[0,1], \quad[1,2] \times[1,2] \times[1,2], \\
[0,1] \times[0,1] \times[1,2],[1,2] \times[1,2] \times[0,1]\}\end{array}$ & \{\} \\
\hline 3 & {$[0,1] \times[1,2] \times[0,1] / \sim$} & $\begin{array}{r}\{[1,2] \times[0,1] \times[1,2],[0,1] \times[1,2] \times[1,2], \\
[0,1] \times[1,2] \times[0,1],[1,2] \times[0,1] \times[0,1]\}\end{array}$ & \{\} \\
\hline 2 & {$[0,0] \times[0,1] \times[0,1] / \sim$} & $\begin{aligned} & \{[0,0] \times[0,1] \times[0,1] \\
& {[1,1] \times[1,2] \times[1,2] } \\
& {[2,2] \times[0,1] \times[0,1]\} }\end{aligned}$ & $\begin{array}{r}\{[0,0] \times[1,2] \times[0,1] \\
{[1,1] \times[0,1] \times[1,2]} \\
[2,2] \times[1,2] \times[0,1]\}\end{array}$ \\
\hline 2 & {$[0,0] \times[0,1] \times[1,2] / \sim$} & $\begin{array}{r}\{[0,0] \times[0,1] \times[1,2], \\
{[1,1] \times[1,2] \times[0,1]} \\
[2,2] \times[0,1] \times[1,2]\}\end{array}$ & $\begin{array}{r}\{[0,0] \times[1,2] \times[1,2], \\
{[1,1] \times[0,1] \times[0,1]} \\
[2,2] \times[1,2] \times[1,2]\}\end{array}$ \\
\hline 2 & {$[0,1] \times[0,0] \times[0,1] / \sim$} & $\begin{array}{r}\{[0,1] \times[0,0] \times[0,1] \\
{[0,1] \times[2,2] \times[0,1]} \\
[1,2] \times[1,1] \times[0,1]\}\end{array}$ & $\begin{array}{r}\{[1,2] \times[0,0] \times[1,2] \\
{[1,2] \times[2,2] \times[1,2]} \\
[0,1] \times[1,1] \times[1,2]\}\end{array}$ \\
\hline 2 & {$[0,1] \times[0,0] \times[1,2] / \sim$} & $\begin{array}{r}\{[0,1] \times[0,0] \times[1,2] \\
{[0,1] \times[2,2] \times[1,2]} \\
[1,2] \times[1,1] \times[1,2]\}\end{array}$ & $\begin{array}{l}\{[1,2] \times[0,0] \times[0,1], \\
{[1,2] \times[2,2] \times[0,1]} \\
[0,1] \times[1,1] \times[0,1]\}\end{array}$ \\
\hline & {$[0,1] \times[0,1] \times[0,0] / \sim$} & $\begin{array}{c}\{[0,1] \times[0,1] \times[1,1] \\
{[0,1] \times[0,1] \times[2,2]} \\
[0,1] \times[0,1] \times[0,0]\}\end{array}$ & $\begin{array}{r}\{[1,2] \times[1,2] \times[1,1], \\
{[1,2] \times[1,2] \times[2,2]} \\
[1,2] \times[1,2] \times[0,0]\}\end{array}$ \\
\hline 2 & {$[0,1] \times[1,2] \times[0,0] / \sim$} & $\begin{array}{r}\{[0,1] \times[1,2] \times[1,1] \\
{[0,1] \times[1,2] \times[2,2]} \\
[0,1] \times[1,2] \times[0,0]\}\end{array}$ & $\begin{array}{r}\{[1,2] \times[0,1] \times[1,1] \\
{[1,2] \times[0,1] \times[2,2]} \\
[1,2] \times[0,1] \times[0,0]\}\end{array}$ \\
\hline 1 & {$[0,0] \times[0,0] \times[0,1] / \sim$} & $\begin{array}{l}\{[2,2] \times[2,2] \times[0,1],[2,2] \times[0,0] \times[0,1] \\
{[1,1] \times[1,1] \times[1,2], \quad[0,0] \times[2,2] \times[0,1],} \\
[0,0] \times[0,0] \times[0,1]\}\end{array}$ & $\begin{array}{l}\{[2,2] \times[1,1] \times[0,1] \\
{[1,1] \times[2,2] \times[1,2]} \\
{[1,1] \times[0,0] \times[1,2]} \\
[0,0] \times[1,1] \times[0,1]\}\end{array}$ \\
\hline 1 & {$[0,0] \times[0,0] \times[1,2] / \sim$} & $\begin{array}{l}\{[0,0] \times[0,0] \times[1,2],[2,2] \times[2,2] \times[1,2] \\
{[0,0] \times[2,2] \times[1,2],[2,2] \times[0,0] \times[1,2]} \\
[1,1] \times[1,1] \times[0,1]\}\end{array}$ & $\begin{array}{r}\{[1,1] \times[2,2] \times[0,1],[1,1] \times[0,0] \times[0,1] \\
[2,2] \times[1,1] \times[1,2],[0,0] \times[1,1] \times[1,2]\}\end{array}$ \\
\hline & {$[0,0] \times[0,1] \times[0,0] / \sim$} & $\begin{array}{c}\{[0,0] \times[0,1] \times[2,2],[0,0] \times[0,1] \times[0,0] \\
{[2,2] \times[1,2] \times[1,1], \quad[2,2] \times[0,1] \times[0,0]} \\
[0,0] \times[1,2] \times[1,1],[2,2] \times[0,1] \times[2,2]\}\end{array}$ & $\begin{array}{r}\{[1,1] \times[1,2] \times[2,2] \\
{[1,1] \times[1,2] \times[0,0]} \\
[1,1] \times[0,1] \times[1,1]\} \\
\end{array}$ \\
\hline & {$[0,0] \times[0,1] \times[1,1] / \sim$} & $\begin{array}{r}\{[0,0] \times[1,2] \times[2,2], \quad[0,0] \times[1,2] \times[0,0] \\
{[2,2] \times[0,1] \times[1,1], \quad[2,2] \times[1,2] \times[0,0],} \\
[0,0] \times[0,1] \times[1,1], \quad[2,2] \times[1,2] \times[2,2]\}\end{array}$ & $\begin{array}{r}\{[1,1] \times[0,1] \times[2,2] \\
{[1,1] \times[0,1] \times[0,0]} \\
[1,1] \times[1,2] \times[1,1]\}\end{array}$ \\
\hline & {$[0,1] \times[0,0] \times[0,0] / \sim$} & $\begin{array}{c}\{[1,2] \times[2,2] \times[2,2],[0,1] \times[2,2] \times[2,2], \\
{[1,2] \times[2,2] \times[0,0], \quad[0,1] \times[0,0] \times[0,0],} \\
{[1,2] \times[0,0] \times[2,2], \quad[0,1] \times[2,2] \times[0,0],} \\
[0,1] \times[0,0] \times[2,2], \quad[1,2] \times[0,0] \times[0,0]\}\end{array}$ & \{\} \\
\hline 1 & {$[0,1] \times[0,0] \times[1,1] / \sim$} & $\begin{array}{r}\{[0,1] \times[0,0] \times[1,1], \quad[1,2] \times[2,2] \times[1,1] \\
[1,2] \times[0,0] \times[1,1], \quad[0,1] \times[2,2] \times[1,1]\}\end{array}$ & $\begin{array}{c}\{[0,1] \times[1,1] \times[0,0],[1,2] \times[1,1] \times[0,0], \\
[1,2] \times[1,1] \times[2,2],[0,1] \times[1,1] \times[2,2]\}\end{array}$ \\
\hline 0 & {$[0,0] \times[0,0] \times[0,0] / \sim$} & $\{15$ points $\}$ & \{\} \\
\hline & {$[0,0] \times[0,0] \times[1,1] / \sim$} & $\{12$ points $\}$ & \{\} \\
\hline
\end{tabular}

Table 1. Cubical cell complex for $Y$. For each equivalence class, the cubical cells with the same and opposite orientation are shown. 
cochains for the cohomology classes of interest. The generator of $\mathbb{Z}_{2} \subset H^{2}\left(Y_{0}, \mathbb{Z}\right)$ can be chosen to be the 2-cochain

$$
\begin{aligned}
c_{0}=\chi & ([0,0] \times[0,0] \times[0,1] \times[0,1] / \sim) \\
& -\chi([0,0] \times[0,0] \times[1,2] \times[0,1] / \sim) .
\end{aligned}
$$

By the retraction property, the 4 -torsion part $\mathbb{Z}_{4} \times \mathbb{Z}_{4} \subset H^{2}\left(Y_{0}, \mathbb{Z}\right)$ is necessarily the pullback of $H^{2}(Y, \mathbb{Z})$. Hence the generators are

$$
\begin{aligned}
c_{1}=\chi & ([0,1] \times[0,1] \times[0,0] \times[0,0] / \sim), \\
c_{2}=\chi([0,0] \times[0,1] \times[0,1] \times[0,0] / \sim) & \\
& +\chi([0,1] \times[0,0] \times[1,2] \times[0,0] / \sim),
\end{aligned}
$$

see eq. (4.12). Finally, a free $\mathbb{Z} \subset H^{2}\left(Y_{0}, \mathbb{Z}\right)$ is generated by

$$
\begin{aligned}
c_{3}=\chi & ([0,1] \times[0,0] \times[0,0] \times[0,1] / \sim) \\
& +\chi([0,1] \times[0,0] \times[1,1] \times[0,1] / \sim)
\end{aligned}
$$

The code producing these calculations and the worksheet can be found in the appendix.

\subsection{Cohomology of the Calabi-Yau manifold}

We know already by eq. (4.4) that $H^{*}\left(Y_{0}, \mathbb{Z}\right)$ is a direct summand of the cohomology of the Calabi-Yau manifold $X$. As far as cup products are concerned, this is all that we will be using in the following. However, for completeness let us note that the entire cohomology group can be computed numerically from the cubical cell complex, and the result is

$$
H^{d}(X, \mathbb{Z})= \begin{cases}\mathbb{Z} & d=6 \\ \mathbb{Z}_{4}^{2} \oplus \mathbb{Z}_{2}^{3} & d=5 \\ \mathbb{Z}^{3} \oplus \mathbb{Z}_{2}^{3} & d=4 \\ \mathbb{Z}^{8} \oplus \mathbb{Z}_{2}^{3} & d=3 \\ \mathbb{Z}^{3} \oplus \mathbb{Z}_{4}^{2} \oplus \mathbb{Z}_{2}^{3} & d=2 \\ 0 & d=1 \\ \mathbb{Z} & d=0 .\end{cases}
$$

If this Calabi-Yau manifold were self-mirror, to the best of our knowledge, this would the first example of a self-mirror Calabi-Yau threefold where the two (a priori independent) torsion groups Torsion $\left(H^{2}(X, \mathbb{Z})\right)=\operatorname{Torsion}\left(H^{5}(X, \mathbb{Z})\right)$ and Torsion $\left(H^{3}(X, \mathbb{Z})\right)=$ Torsion $\left(H^{4}(X, \mathbb{Z})\right)$ actually differ.

\section{Cup product}

\subsection{Cup product on $Y_{0}$}

For orientable manifolds, the cup product is dual to the cap (intersection) product. Now $Y_{0}$ is not orientable, so Poincaré duality does not hold over $\mathbb{Z}$. However, any manifold is $\mathbb{Z}_{2}$-orientable which is sufficient for our purposes since the codomain of the cup product

$$
\cup: H^{2}\left(Y_{0}, \mathbb{Z}\right) \times H^{2}\left(Y_{0}, \mathbb{Z}\right) \rightarrow H^{4}\left(Y_{0}, \mathbb{Z}\right)=\mathbb{Z}_{2}
$$


is two-torsion, anyways. Furthermore, all relevant intersections turn out to be transversal, which lets us read off the cup product from the cochain representatives in eqs. (4.17), (4.12), and (4.19). The result is that

$$
c_{0} \cup c_{1}=c_{2} \cup c_{3} \neq 0
$$

and all other products vanish.

\subsection{Naturality and the Calabi Yau manifold}

Recall that the cup product is natural, that is, the diagram

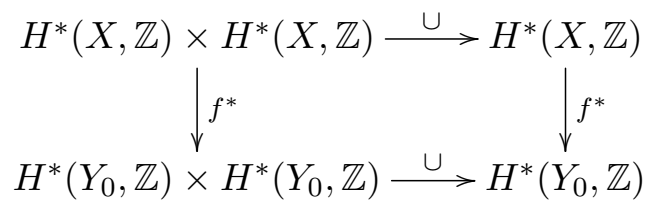

commutes for any map $f: Y_{0} \rightarrow X$. When applied to our embedding map $i: Y_{0} \hookrightarrow X$, we note that $i^{*}$ is surjective by eq. (4.4). In particular, there are elements $\bar{c}_{i} \in H^{2}(X, \mathbb{Z})$ such that $i^{*}\left(\bar{c}_{i}\right)=c_{i}$ are our generators of $H^{2}\left(Y_{0}, \mathbb{Z}\right), i=0,1,2,3$. Their cup products

$$
\bar{c}_{0} \cup \bar{c}_{1}, \bar{c}_{2} \cup \bar{c}_{3} \in H^{4}(X, \mathbb{Z})
$$

must be non-trivial cohomology classes because

$$
i^{*}\left(\bar{c}_{0} \cup \bar{c}_{1}\right)=i^{*}\left(\bar{c}_{2} \cup \bar{c}_{3}\right) \neq 0 \in H^{4}\left(Y_{0}, \mathbb{Z}\right)=\mathbb{Z}_{2}
$$

To summarize, the resulting non-commuting discrete gauge symmetries of four dimensional theory are associated with $\mathbb{Z}_{2} \times \mathbb{Z}_{4}$ sectors of second torsion cohomology and a $\mathbb{Z}_{2}$ sector of the fourth torsion cohomology, resulting in the Heisenberg group determined by $k_{1}=2, k_{2}=4, k_{3}=2$ and $M=1 .^{8}$

\section{Outlook}

In this paper we provided the first explicit example of Type IIB string theory compactification on a Calabi-Yau manifold, which leads to a non-Abelian discrete gauge symmetry in four-dimensions. The compactification is based on the Calabi-Yau threefold whose torsion cohomology structure results in a non-trivial cup product of the second cohomology torsion class elements, thus resulting in a non-Abelian gauge symmetry associated with a Heisenberg-type discrete group.

We should, however, point out that this is a very specific Type IIB compactification on a smooth Calabi-Yau manifold. Generalizations to Calabi-Yau orientifolds, which would also allow for introduction of non-Abelian continuous symmetries and chiral matter, are expected to be straightforward, with generators $\rho_{2}\left(\tilde{\omega}_{4}\right)$ of the second (fourth) cohomology

\footnotetext{
${ }^{8}$ Note that a cup product with the free sector of $H^{2}$ does not result in a non-Abelian discrete symmetry.
} 
torsion classes odd (even) under the orientifold action. ${ }^{9}$ Further studies of such compactifications with a full fledged particle physics content would be an important future research direction.

We should also note that these constructions result in specific non-Abelian discrete gauge symmetries, namely, Heisenberg-type ones. Furthermore, specific results apply here only to four-dimensional field theories, and not to six-dimensional ones, as K3 Calabi-Yau twofolds have no torsion classes. Therefore an important generalization to other CalabiYau manifolds, both twofolds and threefolds, where the non-Abelian discrete symmetries could arise, e.g., from generalized isometries of the compactification manifold, awaits further studies.

Last, but not least, an important direction of this program is to extend studies of nonAbelian discrete gauge symmetries to F-theory, and to shed light on the geometric origins of non-Abelian discrete gauge symmetries there. Techniques spelled out in this paper may open the door for a systematic construction of F-theory compactifications on elliptically fibered Calabi-Yau fourfolds with a structure of torsion cohomologies [19] that would result in four-dimensional non-Abelian discrete symmetries.

\section{Acknowledgments}

We would like to thank Jonathan Block for help with an earlier version of this work, Ling Lin for numerous discussions and a collaboration on related topics, and Angel Uranga for useful communications. The research of Mirjam Cvetič is supported in part by the DOE Grant Award de-sc0013528 (M.C., M.P.), the Fay R. and Eugene L. Langberg Endowed Chair (M.C.) and the Slovenian Research Agency (ARRS) (M.C.). During the preparation of this work Ron Donagi was supported in part by NSF grant DMS 1603526 and by Simons Foundation grant \# 390287.

\section{A Notebook}

The paper describes a six-dimensional Calabi-Yau manifold with a non-trivial cup product between two degree-two torsion classes. As described above, the cup product can be detected by a (non-orientable) four-dimensional submanifold $Y_{0}$, see section 4.2. In this worksheet, we construct a $\Delta$-complex for $Y_{0}$ and determine the cohomology ring structure.

- This worksheet together with all required code can be found on github at https://github.com/vbraun/torsion_cup_product.

- To run this worksheet yourself you need Sage http://sagemath.org and the Python package in the torus_triangulation directory of this repository. The latter is only used to build up the cubical/simplicial $/ \Delta$-complex representation of the $Y_{0}=$ $T^{4} /\left(\mathbb{Z}_{2} \times \mathbb{Z}_{2}\right)$ quotient. The computation of cohomology groups and the cup product is taken from Sage.

\footnotetext{
${ }^{9}$ Note however, that issues related to the manifestly supersymmetric four-dimensional action were raised in $[19]$.
} 
In [3]: from _-future_- import print_function, absolute_import

from sage.all import *

from torus_triangulation.cube_triangulation import CubeTriangulation

from torus_triangulation.builder import TorusQuotientBuilder

\section{A.1 Constructing $Y_{0}$}

Let $z_{i}=x_{i}+\tau y_{i}, i=0, \ldots, 2$ be the three complex coordinates on the 6 -torus covering $Y$, see section 3 of the paper. This worksheet constructs the 4-d submanifold $Y_{0}$ defined by the four coordinates

$$
\left(x_{0}, x_{1}, x_{2}, y_{0}\right)
$$

subject to the identifications:

- The torus $x_{0} \sim x_{0}+2, x_{1} \sim x_{1}+2, x_{2} \sim x_{2}+2$, and $y_{0} \sim y_{0}+1$. Note the different domain on $y_{0}$, which we chose for convenience.

- The $G=\mathbb{Z}_{2} \times \mathbb{Z}_{2}$ group action:

In [4]: def $f$ (point):

return tuple([ point [0]+1, -point[1], $-\operatorname{point[2],~point[3]])}$

def $g$ (point):

return tuple([-point [0], point[1] +1, -point[2] +1, -point[3]])

Up to the $G$-action, we need two unit 4-cubes to cover $Y_{0}$. These are defined below, together with a particular order of their vertices. Combined with a particular choice of triangulation of these unit 4-cubes that is defined by the CubeTriangulation Python class, and as long as everything is invariant under the torus identification and $G$-action, this defines a decomposition into simplicies with an order on the vertices, that is, a $\Delta$-complex.

In fact, most orderings for the vertices clash with the (torus and/or $G$-) identifications and fail to define a $\Delta$-complex. It is a non-trivial fact that there is an ordering that works at all, in general the existence is only guaranteed after subdivision of the simplicies. However, by a computer search we found the following solution:

In $[5]:$ order $1=[$

$$
\begin{aligned}
& (1,1,1,0), \\
& (1,1,1,1), \\
& (0,1,1,0), \\
& (0,1,1,1), \\
& (0,0,0,1), \\
& (0,0,0,0), \\
& (1,0,0,1), \\
& (1,0,0,0), \\
& (0,0,1,1), \\
& (1,0,1,1), \\
& (0,0,1,0), \\
& (1,1,0,0), \\
& (1,0,1,0),
\end{aligned}
$$




$$
\begin{aligned}
& \quad(0,1,0,0), \\
& \quad(1,1,0,1), \\
& \quad(0,1,0,1), \\
& \text { order } 2=[(0[0]+1, \circ[1], 0[2], \circ[3]) \text { for o in order } 1]
\end{aligned}
$$

Given this data, we now build up all simplices on the covering space in the fundamental region $0 \leq x_{0}, x_{1}, x_{2} \leq 2,0 \leq y_{0} \leq 1$

In [6] : builder = TorusQuotientBuilder $(2,2,2,1$, group_gens=[f, g] )

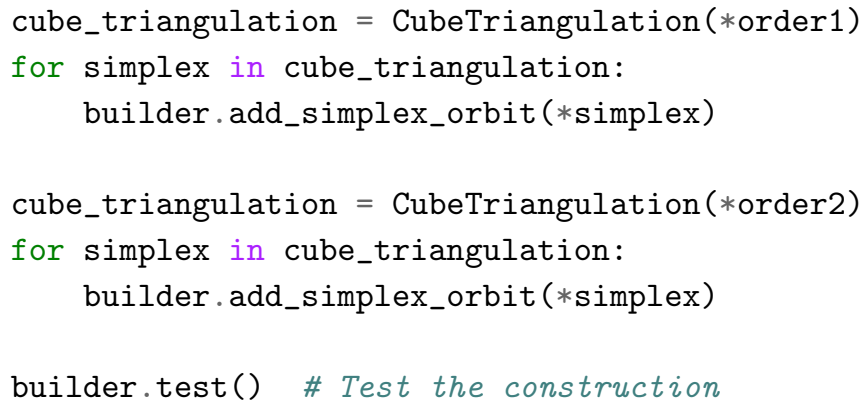

For example, there are 626 two-simplices, the first 5 of which are defined by the vertices In [7]: list (builder.cells.simplices [3] ) [0:5]

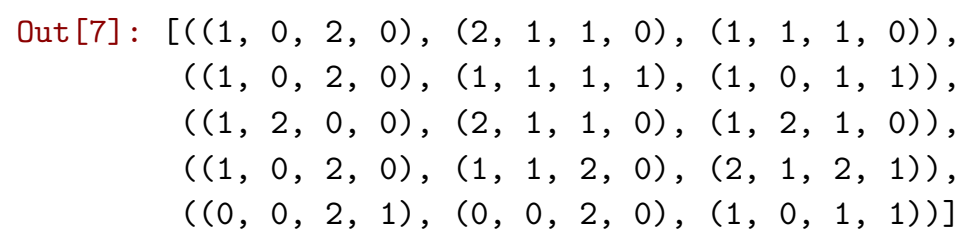

\section{A.2 Cohomology groups: the fundamental region}

As a toy example, consider the $[0,2] \times[0,2] \times[0,2] \times[0,1]$ fundamental region before identifying any cells:

In $[8]:$ cube $=$ builder. cells $\cdot$ delta_complex ()$;$ cube

Out[8]: Delta complex with 54 vertices and 1844 simplices

As expected, it has the cohomology groups of a point:

In [9] : cube. cohomology (reduced=False)

Out $[9]:\{0: Z, 1: 0,2: 0,3: 0,4: 0\}$

\section{A.3 Cohomology groups: four-torus}

Let us check that we indeed obtain a $T^{4}$ by identifying opposing sides (and ignoring the $G$-action):

In $[10]: \mathrm{T} 4=$ builder.torus_cells.delta_complex ()$; \mathrm{T} 4$

Out[10]: Delta complex with 8 vertices and 1201 simplices

In [11]: T4. cohomology (reduced=False)

Out [11]: $\{0: \mathrm{Z}, 1: \mathrm{Z} \times \mathrm{Z} \times \mathrm{Z} \times \mathrm{Z}, 2: \mathrm{Z} \uparrow 6,3: \mathrm{Z} \times \mathrm{Z} \times \mathrm{Z} \times \mathrm{Z}, 4: \mathrm{Z}\}$ 


\section{A.4 Cohomology groups of $Y_{0}$}

Finally, we construct the $\Delta$-complex for the $Y_{0}=T^{4} / G$ quotient

In [12]: Y0 = builder.quotient_cells.delta_complex ()$;$ YO

Out[12]: Delta complex with 2 vertices and 301 simplices

In [13] : Y0. cohomology (reduced=False)

Out [13]: $\{0: \mathrm{Z}, 1: 0,2: \mathrm{Z} \times \mathrm{C} 2 \times \mathrm{C} 4 \times \mathrm{C} 4,3: \mathrm{Z} \times \mathrm{Z}, 4: \mathrm{C} 2\}$

\section{A.5 Cup products}

By dimension, the only interesting case is $H^{2}\left(Y_{0}, \mathbb{Z}\right) \times H^{2}\left(Y_{0}, \mathbb{Z}\right) \rightarrow H^{4}\left(Y_{0}, \mathbb{Z}\right)$. We start by extracting generators in degree two, that is, two-cochains:

In [14]: chains2 = Y0.n_chains (2, base_ring=ZZ, cochains=True)

h2_0, h2_1, h2_2, h2_3 = Y0. cohomology (generators=True, dim=2)

$\mathrm{c0}=$ chains2.from_vector $\left(\mathrm{h} 2 \_0[1] \cdot \operatorname{vector}(2)\right)$

c1 = chains2.from_vector (h2_1[1].vector (2))

c2 = chains2.from_vector(h2_2[1].vector (2))

c3 = chains2.from_vector (h2_3[1].vector (2))

We now verify that the chosen generators of $H^{2}\left(T^{4} / G\right)$ are:

- c0 is two-torsion

- c1, c2 are four-torsion

- c3 is free

In $[15]$ : for $\mathrm{c}$ in $[\mathrm{c0}, \mathrm{c1}, \mathrm{c2}, \mathrm{c3}]$ :

print(c.is_cocycle(), c.is_coboundary(), $(2 * c)$. is_coboundary ()$,(4 * c)$. is_coboundary ()$)$

True False True True

True False False True

True False False True

True False False False

For the codomain of the cup product we also need to chose a generator, which we take to be the four-cochain y0:

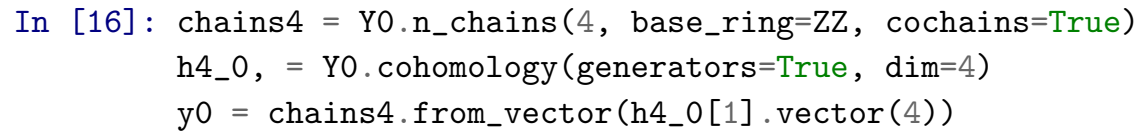

The generator of $H^{4}\left(T^{4} / G\right)$ is y0, and we verify that it is two-torsion:

In [17]: y0.is_cocycle ()$, \mathrm{y} 0$. is_coboundary ()$,(2 * y 0) . i s_{-} \operatorname{coboundary}()$

Out [17]: (True, False, True) 
By checking which degree four expressions are coboundaries, we can build up a list of all cup products:

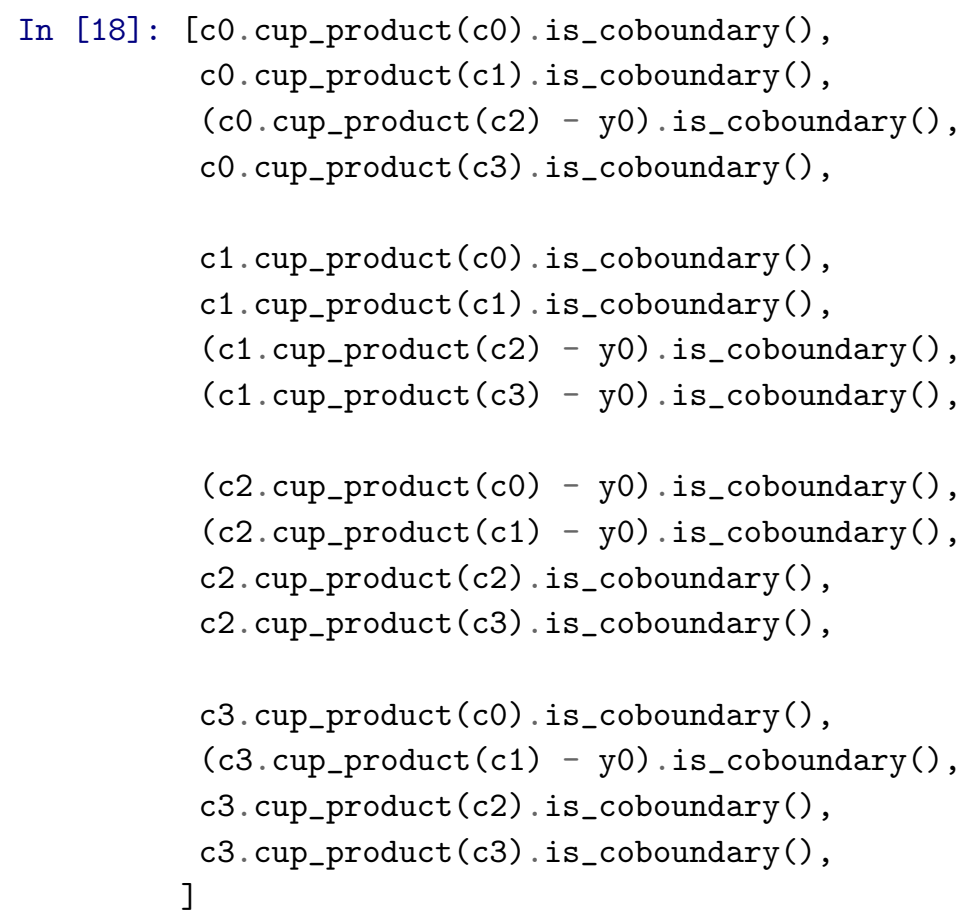

Out [18] : [True,

True,

True,

True,

True,

True,

True,

True,

True,

True,

True,

True,

True,

True,

True,

True]

In [19]: def trivial_cup_product_table(*cohomology_generators):

" " "

Make a table whose entries are whether the cup product

is trivial

" " "

names $=\left[{ }^{\prime} \mathrm{c0} 0^{\prime},{ }^{\prime} \mathrm{c} 1{ }^{\prime},{ }^{\prime} \mathrm{c} 2{ }^{\prime},{ }^{\prime} \mathrm{c}^{\prime}\right]$

rows $=\left[{ }^{\prime},\right]+$ names $]$

for row_name, $c$ in zip(names, cohomology_generators): 


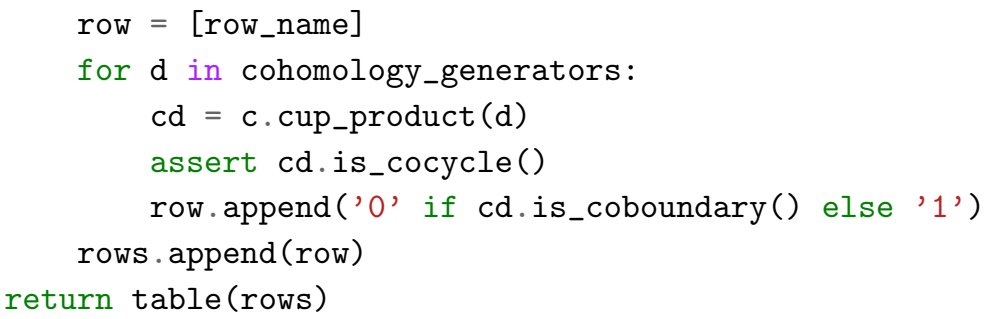

\section{A.6 Alternate basis}

There is a slightly better basis choice that leads to fewer non-trivial table entries:

In [21]: trivial_cup_product_table(c0, c2, c1+c0, c3+2*c2)

Out [21]: $\quad$ c0 $\quad$ c1 $\quad$ c2 $\quad$ c3

c0 $0 \quad 1 \quad 000$

$\begin{array}{lllll}c 1 & 1 & 0 & 0 & 0\end{array}$

c2 $0 \quad 0 \quad 001$

$\begin{array}{lllll}\text { c3 } & 0 & 0 & 1 & 0\end{array}$

Open Access. This article is distributed under the terms of the Creative Commons Attribution License (CC-BY 4.0), which permits any use, distribution and reproduction in any medium, provided the original author(s) and source are credited.

\section{References}

[1] V. Braun and D.R. Morrison, F-theory on Genus-One Fibrations, JHEP 08 (2014) 132 [arXiv:1401.7844] [INSPIRE].

[2] D.R. Morrison and W. Taylor, Sections, multisections and U(1) fields in F-theory, arXiv: 1404.1527 [INSPIRE].

[3] L.B. Anderson, I. García-Etxebarria, T.W. Grimm and J. Keitel, Physics of F-theory compactifications without section, JHEP 12 (2014) 156 [arXiv:1406.5180] [INSPIRE].

[4] D. Klevers, D.K. Mayorga Pena, P.-K. Oehlmann, H. Piragua and J. Reuter, F-Theory on all Toric Hypersurface Fibrations and its Higgs Branches, JHEP 01 (2015) 142 [arXiv: 1408.4808] [INSPIRE].

[5] I. García-Etxebarria, T.W. Grimm and J. Keitel, Yukawas and discrete symmetries in F-theory compactifications without section, JHEP 11 (2014) 125 [arXiv:1408.6448] [INSPIRE].

[6] C. Mayrhofer, E. Palti, O. Till and T. Weigand, Discrete Gauge Symmetries by Higgsing in four-dimensional F-theory Compactifications, JHEP 12 (2014) 068 [arXiv:1408.6831] [INSPIRE]. 
[7] C. Mayrhofer, E. Palti, O. Till and T. Weigand, On Discrete Symmetries and Torsion Homology in F-theory, JHEP 06 (2015) 029 [arXiv: 1410.7814] [INSPIRE].

[8] M. Cvetič, R. Donagi, D. Klevers, H. Piragua and M. Poretschkin, F-theory vacua with $\mathbb{Z}_{3}$ gauge symmetry, Nucl. Phys. B 898 (2015) 736 [arXiv:1502.06953] [INSPIRE].

[9] M. Cvetič, A. Grassi and M. Poretschkin, Discrete Symmetries in Heterotic/F-theory Duality and Mirror Symmetry, JHEP 06 (2017) 156 [arXiv: 1607.03176] [INSPIRE].

[10] M. Berasaluce-Gonzalez, P.G. Camara, F. Marchesano, D. Regalado and A.M. Uranga, Non-Abelian discrete gauge symmetries in 4d string models, JHEP 09 (2012) 059 [arXiv: 1206.2383] [INSPIRE].

[11] P.G. Camara, L.E. Ibáñez and F. Marchesano, RR photons, JHEP 09 (2011) 110 [arXiv:1106.0060] [INSPIRE].

[12] M. Berasaluce-Gonzalez, L.E. Ibáñez, P. Soler and A.M. Uranga, Discrete gauge symmetries in D-brane models, JHEP 12 (2011) 113 [arXiv:1106.4169] [INSPIRE].

[13] L.E. Ibáñez, A.N. Schellekens and A.M. Uranga, Discrete Gauge Symmetries in Discrete MSSM-like Orientifolds, Nucl. Phys. B 865 (2012) 509 [arXiv:1205.5364] [InSPIRE].

[14] S. Gukov, M. Rangamani and E. Witten, Dibaryons, strings and branes in AdS orbifold models, JHEP 12 (1998) 025 [hep-th/9811048] [INSPIRE].

[15] R. Donagi and K. Wendland, On orbifolds and free fermion constructions, J. Geom. Phys. 59 (2009) 942 [arXiv: 0809.0330] [INSPIRE].

[16] J. Igusa, On the structure of a certain class of Kähler varieties, Amer. J. Math. 76 (1954) 669.

[17] K. Ueno, Classification Theory of Algebraic Varieties and Compact Complex Spaces, Lect. Notes Math. 439 (1975) 228.

[18] K. Oguiso and J. Sakurai, Calabi-Yau threefolds of quotient type, Asian J. Math. 5 (2001) 43 [math/9909175].

[19] T.W. Grimm, T.G. Pugh and D. Regalado, Non-Abelian discrete gauge symmetries in F-theory, JHEP 02 (2016) 066 [arXiv:1504.06272] [INSPIRE].

[20] V. Braun, M. Kreuzer, B.A. Ovrut and E. Scheidegger, Worldsheet instantons and torsion curves, part A: Direct computation, JHEP 10 (2007) 022 [hep-th/0703182] [INSPIRE].

[21] A. Hanany and E. Witten, Type IIB superstrings, BPS monopoles and three-dimensional gauge dynamics, Nucl. Phys. B 492 (1997) 152 [hep-th/9611230] [INSPIRE].

[22] C. Vafa and E. Witten, On orbifolds with discrete torsion, J. Geom. Phys. 15 (1995) 189 [hep-th/9409188] [INSPIRE].

[23] K. Hashimoto and A. Kanazawa, Calabi-Yau threefolds of type K (I): Classification, arXiv:1409.7601 [INSPIRE].

[24] R. Donagi and A.E. Faraggi, On the number of chiral generations in Z(2) x Z(2) orbifolds, Nucl. Phys. B 694 (2004) 187 [hep-th/0403272] [InSPIRE].

[25] C. Manolescu, Pin(2)-equivariant Seiberg-Witten Floer homology and the Triangulation Conjecture, J. Amer. Math. Soc. 29 (2016) 147 [arXiv:1303.2354]. 